\title{
A short-duration dark adaptation protocol for assessment of age-related maculopathy
}

\author{
Gregory R. Jackson • John G. Edwards
}

Received: 12 November 2007 / Accepted: 13 February 2008 / Published online: 6 May 2008

(C) Humana Press 2008

\begin{abstract}
Dark adaptometry may be a useful diagnostic test and clinical trial endpoint for age-related maculopathy (ARM) because impaired night vision is a hallmark of early ARM. A novel dark adaptometer, the AdaptDx, was evaluated for the detection of ARM. The AdaptDx incorporates a 20-minute protocol optimized for the detection of ARM. ARM patients $(N=17)$ exhibited substantial dark adaptation impairment compared with normal adults $(N=17)$. The diagnostic sensitivity was $88 \%$ and the specificity was $100 \%$. The diagnostic test characteristics of the AdaptDx are similar to previously reported studies using 60- to 120 -minute protocols.
\end{abstract}

Keywords Age-related macular degeneration .

Macular degeneration - Diagnostic - Clinical trial endpoint .

Dark adaptation $\cdot$ Human

\section{Introduction}

Age-related maculopathy (ARM), also known as agerelated macular degeneration, affects approximately 30 million people worldwide. In the United States, there are

Financial Disclosure: G. Jackson and J. Edwards hold intellectual property related to the AdaptDx. J. Edwards has a significant financial interest in the AdaptDx.

G. R. Jackson $(\bowtie)$

Department of Ophthalmology, Penn State College of Medicine, 500 University Drive, HU19,

Hershey, PA 17033-0850, USA

e-mail: gjackson@psu.edu

J. G. Edwards

Apeliotus Technologies, Inc.,

Atlanta, GA, USA an estimated 13 million people with some vision loss from ARM, including two million with the most severe late-stage of the disease. Fortunately, there are an increasing number of effective treatments for late-stage disease, such as pegaptabnib (Macugen; Pfizer) and ranibizumab (Lucentis; Genetech). However, treatment of advanced ARM often results in less than optimal patient outcomes because moderate to severe irreversible vision loss has already occurred before stabilization. The treatment of the disease in its earliest stages is the best strategy to preserve the patient's vision, which may be only mildly disturbed as measured by high contrast visual acuity measured under high luminance conditions.

There are significant barriers to the development of treatments for early ARM. The disease's slow progression hampers the feasibility of clinical trials because currently accepted endpoints of acuity and fundus appearance are relatively insensitive to the disease's progression through its earliest stages. These endpoints require clinical trials to have large sample sizes or long follow-up durations to evaluate efficacy of a potential treatment. Clinical trials evaluating early treatments are impractical because of the constraints current endpoints impose on trial design. More sensitive endpoints are required to enhance the feasibility of early ARM clinical trials.

While patients with early ARM typically have good best-corrected visual acuity, impaired night vision is a prominent self-reported problem [1-3]. Anatomical studies of donor eyes have documented significant rod dysfunction and photoreceptor drop out in comparison to cone photoreceptors [4]. Histopathological studies have found ARMrelated lesions in the RPE and Bruch's membrane, which are generally invisible to clinical inspection $[5,6]$. Not surprisingly, these insults to the photoreceptors and the RPE, which supports the photoreceptors, disrupt vision. Early ARM patients exhibit moderate to severe impairment 
of rod-mediated dark adaptation and scotopic visual sensitivity even in the absence of visual acuity loss [7, 8]. Rod-mediated dark adaptation appears to be more impaired in early ARM patients than photopic visual sensitivity, scotopic visual sensitivity, acuity, and contrast sensitivity [8]. Advanced ARM patients exhibit impaired conemediated dark adaptation [9-12] and early ARM patients exhibit photostress impairment [13, 14]. However, rodmediated dark adaptation appears to be more affected than cone-mediated dark adaptation at least outside the fovea in early ARM patients [15]. Rod-mediated dark adaptation impairment is modestly reversible with vitamin A supplementation, whereas cone-mediated dark adaptation impairment is not [2]. Impairment of rod-mediated dark adaptation is related to the disease severity [8]. A pilot retrospective study suggests that the rod-mediated dark adaptation impairment is detectable at least 4 years before the lesions associated with the disease are clinically apparent (unpublished data). These findings suggest that dark adaptation impairment is a sensitive marker of early ARM and raises the possibility that it may used to predict progression.

The utility of dark adaptometry as a clinical outcome measure or practical diagnostic tool is hampered by a long test duration, high participant burden, and lack of standardized dark adaptometers. Dark adaptation protocols used in prior research require up to 90 minutes, and typically more than 100 threshold estimates are made. The long duration and number of thresholds measurements may fatigue some patients and aversively affect reliability. These limitations prevent the inclusion of dark adaptometry in clinical trials.

The goal of this study was to develop a short-duration dark adaptation protocol that minimized patient fatigue, increased operator ease of use, and maintained the high sensitivity and specificity of research protocols. The protocol evaluated in this study has three distinct differences compared with prior research protocols: (1) the bleaching light intensity was significantly reduced to shorten the duration necessary to measure sensitivity recovery; (2) the stimulus was located within the area of greatest rod dysfunction to enhance the sensitivity of the test for detection of early disease; and (3) the speed of dark adaptation was estimated using a parameter that is robust to the shape of the dark adaptation function, which can be variable with disease severity. This protocol was implemented using a new dark adaptometer, the AdaptDx, developed in collaboration with Apeliotus Technologies, Inc. (Atlanta, GA). This study evaluated the sensitivity and specificity of the protocol.

\section{Materials and methods}

The Institutional Review Board of the University of Alabama at Birmingham approved this study. Written informed consent was obtained from all participants after the nature and possible consequences of the study were explained. Normal adults and early to intermediate ARM patients were recruited from the comprehensive ophthalmology and retina services of the Department of Ophthalmology, University of Alabama at Birmingham.

Inclusion criteria were as follows: (1) participants were 20 to 45 years old for the young normal group and participants were at least 55 years of age for the old normal and ARM groups; and (2) best-corrected distance visual acuity of 20/100 or better in at least one eye. If both eyes qualified, the eye with the better acuity was enrolled as the test eye. If the acuities were the same, the right eye was enrolled as the test eye.

Exclusion criteria were as follows: (1) medical record or a general health interview indication of glaucoma, optic neuropathy or any ocular conditions other than ARM, or refractive error (spherical equivalent) having an absolute value $>6$ diopters; (2) neurological diseases such as Alzheimer's disease, Parkinson's disease, history of stroke or multiple sclerosis; (3) diabetes; and/or (4) inability to perform the psychophysical task used to measure dark adaptation.

Best-corrected distance visual acuity was measured for each eye using the EDTRS chart and expressed as logMAR. Dark adaptation was measured using the AdaptDx, a computer-automated dark adaptometer. Figure 1 shows the patient's view. The patient's test eye is dilated to $\geq 6 \mathrm{~mm}$ diameter, and corrective lenses are introduced as appropriate for the 30-cm viewing distance to correct for blur. The fellow eye is occluded with an eye patch. An infrared camera located behind the fixation light continuously monitors the patient's test eye and displays an image of the eye on a personal computer-based operator control screen. The

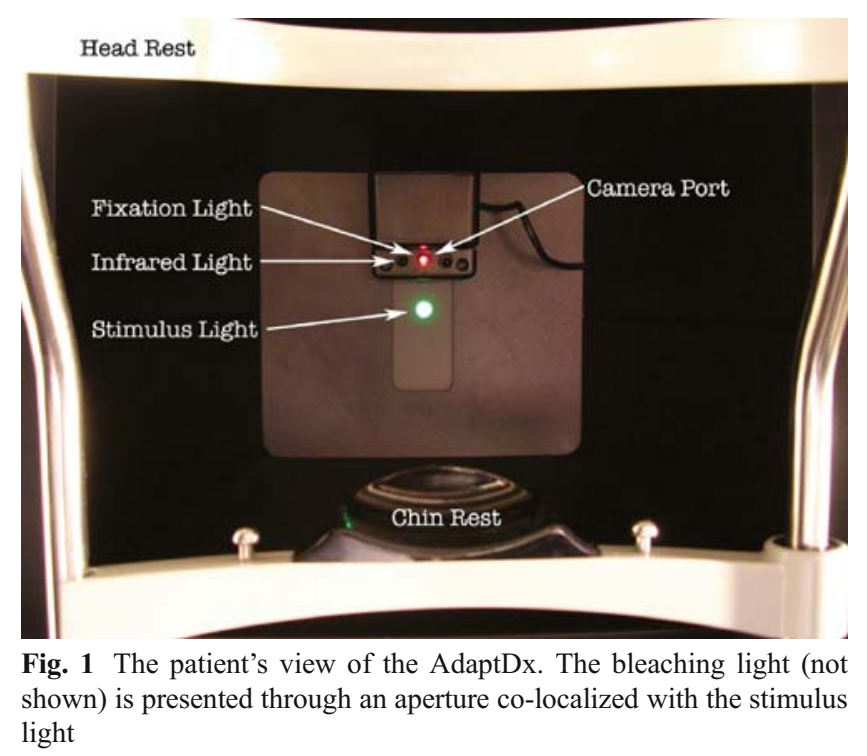


operator centers the patient's test eye to the red fixation light with the help of a reticule displayed on the image of the eye. The patient's test eye is bleached by exposure to a photoflash (0.25 ms duration, $6.38 \log$ scot $\mathrm{Td} \mathrm{second}^{-1}$ intensity) while the patient is focused on the fixation light. The flash of light passes through a diffuser and $4^{\circ}$-diameter aperture centered at $5^{\circ}$ on the inferior visual meridian to provide a uniform focal bleach surrounding the area to be tested during sensitivity recovery measurements. Sensitivity measurements begin immediately after bleach offset. The patient focuses on the fixation light, and responds that a stimulus light is present by pushing a button. The stimulus light is a $1.7^{\circ}$ circular test spot located at $5^{\circ}$ on the inferior visual meridian. To focus on rod-mediated function, a stimulus wavelength of $500 \mathrm{~nm}$ was used, which is near the peak of rod sensitivity. Sensitivity is estimated using a three-down/one-up modified staircase threshold estimate procedure. Starting at a relatively high intensity $\left(5.00 \mathrm{~cd} / \mathrm{m}^{2}\right)$, stimulus lights are presented every two or three seconds for a $200-\mathrm{ms}$ duration. If the patient does not respond within 2 seconds of stimulus onset, the stimulus light intensity remains unchanged on successive stimulus presentations until the patient responds. If the patient indicates that the stimulus is visible, the intensity is decreased for each successive presentation in steps of $0.3 \log$ units until the patient stops responding that the stimulus is visible. After the patient indicates that the stimulus light is invisible by not pressing the button while the stimulus light is present, the intensity of the target is increased for each successive presentation in $0.1 \log$ unit steps until the patient responds that the stimulus light is once again visible. This intensity is defined as a threshold. Successive threshold measurements start with the stimulus intensity $0.2 \log$ units brighter than the previous threshold measurement. The patient has a 30-second rest period between threshold measurements. Threshold measurements are made about once a minute for the duration of the measurement protocol; thus about 20 threshold measurements are made during the 20-minute test.

Because ARM patients can exhibit several differently shaped dark adaptation functions, the "rod intercept", an estimate of recovery speed that is robust to shape, was used. The rod intercept is the amount of time after bleach offset required for the patient's sensitivity to recover to a stimulus intensity of $5 \times 10^{-4} \mathrm{~cd} / \mathrm{m}^{2}$ (i.e.; $4.0 \mathrm{log}$ units). This light level was chosen because it typically is achieved late in the second component of rod-mediated dark adaptation and is therefore completely mediated by rod function. Each subject's dark adaptation function is plotted and the rod intercept is estimated by linear interpolation. Patients with normal rod recovery will have shorter rod intercepts than those with impaired dark adaptation.

Stereoscopic $30^{\circ}$ photographs were taken with a FF450 Plus Zeiss fundus camera for all participants more than 54 years old. Photographs were evaluated using the Agerelated Eye Disease Study (AREDS) Severity Scale for Age-Related Macular Degeneration [16] by two independent graders who were masked to the clinical and functional characteristics of the participants. Group assignment to normal or ARM was based on the AREDS severity score of the test eye. These steps are indicative of normal (step 1), borderline ARM (our terminology) (step 2), early ARM (steps 3-6), intermediate ARM (steps 7-9), and advanced ARM (steps 10 and 11).

\section{Results}

The study population consisted of eight normal young adults (mean age $=32.6$ years old), nine normal old adults (mean age $=73.1$ years old) and 17 ARM patients (mean age $=75.1$ years old). The old normal and ARM groups had similar ages $(p=0.42)$ and test eye acuities $(p=0.14)$ (Table 1$)$.

Table 1 Participant demographics

\begin{tabular}{lccc}
\hline & Young Normal $(N=8)$ & Old Normal $(n=9)$ & ARM $(n=17)$ \\
\hline Age (years), mean (SD) & $32.6(5.3)$ & $73.1(4.8)$ & $75.1(6.4)$ \\
Gender, \% $(n)$ & $100(8)$ & $66.7(6)$ & $52.9(9)$ \\
Female & $0(0)$ & $33.3(3)$ & $47.1(8)$ \\
Male & & & \\
Race, \% $(n)$ & $62.5(5)$ & $88.9(8)$ & $11.1(1)$ \\
White & $25.0(2)$ & $0(0)$ & $0(17)$ \\
African American & $12.5(1)$ & $.04(.12)$ & $0(0)$ \\
Asian & & $.14(.21)$ & $.14(.19)$ \\
Visual acuity, logMAR mean (SD) & $-.02(0.4)$ & $.52(.40)$ \\
Tested eye & $.05(0.1)$ & & \\
Fellow eye &
\end{tabular}




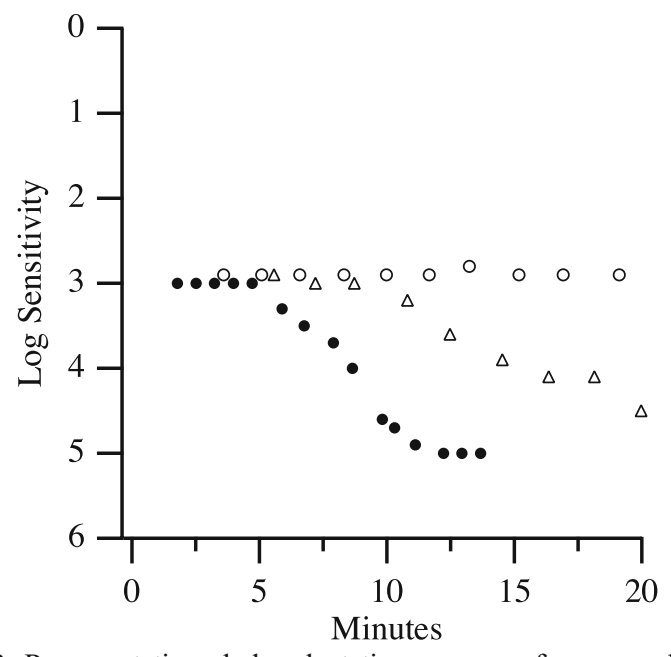

Fig. 2 Representative dark adaptation curves of a normal adult (closed circles), early ARM patient (open triangles), and intermediate ARM patient (open circles)

Fellow eye acuity was higher on average for old normal adults than ARM patients $(p=0.01)$. ARM patients exhibited substantially slower dark adaptation compared with normal old adults; whereas the dark adaptation speed was essentially the same for the young and old normal participants. Representative dark adaptation functions for a young normal adult, an early ARM patient and an intermediate ARM patient are shown in Fig. 2. The rod intercept for ARM patients (mean=17.20 $\mathrm{min}, \mathrm{SD}=3.8$ ) was on average twice as long as the rod intercept for normal old adults (mean= $8.73 \mathrm{~min}, \mathrm{SD}=1.9) \quad(p<0.0001)$. Nine of the $17 \mathrm{ARM}$ patients failed to obtain the criterion sensitivity within the 20-minute protocol, and were assigned a rod intercept of $20 \mathrm{~min}$; thus the substantial difference between these averages actually understates the relative impairment between the two groups. The rod intercept for normal young adults (mean=8.2 min, $\mathrm{SD}=1.4$ ) was statistically indistinguishable from normal old adults $(p=0.76)$.

Each subject's dark adaptation was classified as normal or impaired based whether the participant's speed of dark adaptation as measured by the rod intercept fell within the normal reference range (mean $\pm 2 \mathrm{SD}$ ) of the normal old adults. The upper limit of the normal reference range was 12.5 minutes. Individuals with rod intercepts longer than 12.5 minutes were classified as having impaired dark adaptation. Participants with a rod intercept $\leq 12.5$ were classified as having normal dark adaptation. Using this criterion, 15 of the 17 ARM patients were classified as having abnormal dark adaptation for a diagnostic sensitivity of $88 \%$. All nine normal adults fell within the normal reference range for a diagnostic specificity of $100 \%$. The sensitivity and specificity of our 20-minute study protocol compares favorably to that of a previously used 90-minute research protocol ( $85 \%$ sensitivity; $100 \%$ specificity) [8], despite the considerable shortening of the test. Furthermore, it is useful to examine the two false negative results (those ARM patients classified with normal dark adaptation). Both were for participants with an AREDS fundus grade of 2 . One of these patients exhibited three drusen of $63 \mu \mathrm{m}$ scattered throughout the central 3,000 $\mu \mathrm{m}$ of the macula. The other patient had a small amount of hyperpigmentation surrounding a single $63-\mu \mathrm{m}$ druse. The sensitivity of the test is $100 \%$ for those ARM patients with an AREDS grade 3 and higher.

As shown in Table 2, dark adaptation impairment increased with ARM severity. Old normal adults had a mean rod intercept of $8.7 \mathrm{~min}$. The rod intercept for borderline ARM patients (AREDS fundus grade 2) was, on average, 5.1 minutes slower than the normal old adult's rod intercept. The rod intercept for early ARM patients (AREDS fundus grades 3 to 5) was, on average, yet another 3.4 minutes slower than borderline ARM patients. Finally, all of the intermediate and late ARM patients (AREDS fundus grades $\geq 6$ ) failed to reach the rod intercept within the 20-minute test time.

\section{Conclusions}

Using a 20-minute, rod-mediated dark adaptation protocol, ARM patients exhibited a dramatic slowing of dark adaptation in comparison with normal old adults. The magnitude of the impairment was similar to that previously measured using a 90-minute research protocol [8]. As expected, reducing the bleaching light shortened the duration of the test, and moving the stimulus to an area of greater rod dysfunction preserved the sensitivity and specificity of the protocol. The magnitude of the dark

Table 2 Dark adaptation impairment increased with disease severity

\begin{tabular}{lllllll}
\hline & Young Normal & Old Normal & Borderline ARM & Early ARM & Intermediate ARM & Advanced ARM \\
\hline Sample Size & 8 & 9 & 5 & 6 & 5 & 1 \\
AREDS Fundus Grade & $\mathrm{N}^{\mathrm{a}} \mathrm{A}^{\mathrm{a}}$ & 1 & 2 & $3,4,5$ & $6,7,8,9$ & $\geq 10$ \\
Rod Intercept, min mean (SD) & $8.2(1.4)$ & $8.7(1.9)$ & $13.8(4.4)$ & $17.2(2.7)$ & $20\left({ }^{\mathrm{b}}\right)$ & $20\left({ }^{\mathrm{b}}\right)$ \\
\hline
\end{tabular}

${ }^{a}$ Fundus photography and grading was not performed on young normal group.

${ }^{\mathrm{b}}$ All subjects failed to reach the rod intercept within the 20-minute test duration. Rod intercepts were set at 20 minutes for comparison purposes. 
adaptation impairment exhibited by early ARM patients suggests that a rapid diagnostic screening test having a duration of less than 10 minutes is feasible. Test-retest reliability was found to be quite high (intraclass $r=0.95$ ) in a separate, preliminary study of 40 normal adults and ARM patients (data not shown). Perhaps more intriguing, the increase in the amount of rod-mediated dark adaptation impairment with increasing disease severity as assessed by the AREDS Severity System for ARM allows the possibility of using dark adaptation as a clinical outcome measure.

Previously, we evaluated another short-duration protocol developed for the SST-1 dark adaptometer (LKC Technologies, Inc) for the detection of early ARM [17]. The SST-1 is a manual dark adaptometer that uses an abbreviated protocol, typically 20 to 30 minutes. The AdaptDx and the SST-1 exhibit markedly different response characteristics. Whereas the AdaptDx shows no difference with aging but is highly sensitive to ARM, the SST-1 discriminates aging effects but is insensitive to ARM. An important difference between the protocols is that the SST-1 measures the sensitivity recovery of the entire retina, instead of just the macula or a portion of the macula. Healthy peripheral retina probably masks the dark adaptation impairment localized to the macula.

We have developed a short duration protocol that can sensitively and specifically detect early ARM. Night vision is the first casualty of ARM. The impaired night vision encountered in early ARM is a clinically significant problem similar to acuity impairment encountered in late ARM. As such, we believe dark adaptation is a suitable primary endpoint to evaluate efficacy of treatments aimed at early ARM.

Acknowledgments This work was supported in part by Grant Number R43AG026222 from the National Institute on Aging. The content is solely the responsibility of the authors and does not necessarily represent the official views of the National Institute on Aging or the National Institutes of Health. Additional support provided by the International Retinal Research Foundation, EyeSight Foundation of Alabama, and Research to Prevent Blindness.

\section{References}

1. Scilley K, et al. Early age-related maculopathy and self-reported visual difficulty in daily life. Ophthalmology 2002;109:12351242.

2. Owsley C, et al. Effect of short-term, high-dose retinol on dark adaptation in aging and early age-related maculopathy. Invest Ophthalmol Vis Sci 2006;47(4):1310-8.

3. Owsley C, et al. Development of a questionnaire to assess vision problems under low luminance in age-related maculopathy. Invest Ophthalmol Vis Sci 2006;47(2):528-35.

4. Curcio CA, Medeiros NE, Millican LC. Photoreceptor loss in agerelated macular degeneration. Invest Ophthalmol Vis Sci 1996;37 (7):1236-1249.

5. Curcio CA, Millican CL. Basal linear deposit and large drusen are specific for early age-related maculopathy. Arch of Ophthalmol 1999;117:329-339.

6. Curcio CA, et al. Accumulation of cholesterol with age in human Bruch's membrane. Invest Ophthalmol Vis Sci 2001;42:265-274.

7. Owsley C, et al. Psychophysical evidence for rod vulnerability in age-related macular degeneration. Invest Ophthalmol \& Vis Sci 2000;41:267-273.

8. Owsley C, et al. Delays in rod-mediated dark adaptation in early age-related maculopathy. Ophthalmology 2001;108:1196-1202.

9. Brown B, Adams AJ, Coletta NJ. Dark adaptation in age-related maculopathy. Ophthalmic Physiol Opt 1986;6(1):81-84.

10. Brown B, et al. Cone adaptation in age-related maculopathy. Am J Optom Physiol Opt 1985;63(6):450-454.

11. Brown B, et al. Cone adaptation in age-related maculopathy. Am J Optom Physiol Opt 1986;63(6):450-4.

12. Phipps JA, Guymer RH, Vingrys AJ. Loss of cone function in age-related maculopathy. Invest Ophthalmol Vis Sci 2003;44 (5):2277-2283.

13. Midena E, et al. Macular function impairment in eyes with early age-related macular degeneration. Invest Ophthalmol Vis Sci 1997;38(2):469-77.

14. Binns AM, Margrain TH. Evaluating retinal function in agerelated maculopathy with the ERG photostress test. Invest Ophthalmol Vis Sci 2007;48(6):2806-13.

15. Owsley $\mathrm{C}$, et al. Cone- and rod-mediated dark adaptation impairment in age-related maculopathy. Ophthalmology 2007;114:1728-1735.

16. Davis MD, et al. The Age-Related Eye Disease Study severity scale for age-related macular degeneration: AREDS Report No. 17. Arch Ophthalmol 2005;123(11):1484-98.

17. Jackson GR, Felix T, Owsley C. The Scotopic Sensitivity Tester-1 and the detection of early age-related macular degeneration. Ophthalmic Physiol Opt 2006;26:431-437. 